\title{
An empirical investigation of memory for routes through menu structures
}

\author{
Juliet Richardson, Andrew Howes and Stephen J. Payne \\ School of Psychology, University of Wales, College of Cardiff. \\ PO Box 901, Cardiff, CF1 3YG, U.K. \\ RichardsonJ@Cardiff.ac.uk
}

\begin{abstract}
Menu structures are a pervasive mechanism for interacting with computers. However, whilst a number of investigators have studied the effects of different types of menu structure on initial search performance, there is little empirical work on how routes experienced through exploration, are committed to memory. This paper reports a preliminary empirical investigation of human memory for this kind of skill. Specific predictions are derived from a functional model, called AYN (Howes, 1994). The predictions of the model and the actual performance of the subjects are compared and the implications of the findings for the model are discussed.
\end{abstract}

KEYWORDS Cognitive Models, Computer Menus, Interactive Search, Displays, Recognition Memory.

\section{INTRODUCTION}

We report a preliminary experiment designed to investigate memory for routes through menu structures. We are particularly interested in how subjects remember routes that have been experienced during exploration. An understanding of memory for this kind of experience could be useful in designing a wide range of interactive systems including world wide web pages and browsers.

Several researchers have studied human exploratory search of menus, and a number of important observations have been made. Most of these relate to the strategies used in the search process rather than to memory for this process, but nonetheless they help uncover the nature of what needs to be remembered.

The most immediate and important observation is that menu search is semantically guided. Empirical investigations of subjects learning to use various versions of the CA Cricket Graph and MS EXCEL graphing packages have been carried out (Franzke, 1994; Franzke, 1995). Subjects were set tasks such as "change the line and symbol style of the plotted graph," some of which involved the use of menu structures. Response times were measured for menu labels that (a) were also words in the task description; (b) were synonyms with words in the task description; (c) were semantically related to the task and (d) had no link at all to the task. Response times during an initial exploratory trial were found to be fastest for the overlapping words and slowest for the words that had no links at all. These differences were reduced with practice.

Of course, in real applications it is rarely the case that the everyday semantics of labels are sufficient to guide the novice user through the correct route. In such circumstances, users have to combine semantic guided search with less knowledge-dependent and more general purpose search strategies. In situations where the semantics are not sufficient, subjects using Cricket Graph have been observed to manage search by using an "iterative deepening" procedure: subjects search both the external world defined by the menu structure and an internal world of possible interpretations of menu labels (Rieman, 1994). A model, IDXL, of "iteratively deepening" exploratory search has been developed (Rieman, Young and Howes, 1996). A central mechanism is that for any selection the model must make a choice as to whether to search the internal world

Human-Computer Interaction: INTERACT'97 S. Howard, J. Hammond \& G. Lindgaard (editors) Published by Chapman \& Hall OIFIP 1997 
of knowledge, or the external world of the device. If it decides to search the internal world then it elaborates its semantic interpretation of the visible menu labels. If it decides to search the external world then it chooses the item that is judged most likely to lead to the goal. This item may be on a menu other than the current menu.

Rieman et al.'s model is of novice behaviour, which is obviously different from the behaviour of experts who will have extensive knowledge of menu functions to guide their search and who should therefore rely less on strategies such as semantic guidance and "iterative deepening". Kitajima and Polson (1995) report a model of skilled use of the Cricket Graph package. The model uses representations of the user's goals and of the task environment. These representations are linked by an action cycle consisting of two stages: an evaluation stage and an execution stage. During the evaluation stage the model generates a representation of the objects present in the display. This representation only contains limited information on object appearance. The model then elaborates this representation by a memory sampling process using the goals and the display objects as cues for retrieval from long term memory. During this process information is retrieved about the functions of objects and their links with the goals and with the actions to be performed. For example, the initial representation of a menu item might be elaborated to include knowledge that the menu item can be pulled down as well as information about what items will be present on that pull-down menu. This elaboration process is probabilistic and even with all of the relevant information present in long term memory the model may fail to elaborate the display representation fully leading to errors as a result of incomplete information. During the second phase of the cycle, the execution stage, three candidate objects from the display are selected and the possible actions that can be carried out on them are considered. Eventually one action-object pair is selected and that action is executed on the selected object thus changing the task environment and restarting the action cycle.

The two models described above are candidate models of how experts and novices search during exploration but they leave open the question of how memories are encoded during the search process. How do people perform a menu search task for the second, third or fourth time? How does performance improve?

Howes (1994) reports a functional model, called AYN, of the exploratory acquisition of menu knowledge. AYN acquires two types of knowledge as it interacts with a menu structure: recognition knowledge and control knowledge.

In AYN, recognition knowledge consists of episodic chunks that are encoded into memory for every combination of goal, menu and action that the model experiences regardless of whether the action leads to the goal. AYN also acquires recognition knowledge that the goal has been achieved. Recognition knowledge, of course, does not directly support recall, merely identification of the menus that have been previously visited, which selections made and which goals visited.

AYN uses recognition knowledge to help guide search in the menu structure both during initial exploration and during subsequent trials. A set of rules determines how the model applies this knowledge: (1) if the goal has not yet been achieved then avoid recognised selections; (2) if the goal has been achieved and there is a recognised selection then it should be applied; (3) if there are no recognised selections and the goal has been achieved then AYN should apply a backup operator. These rules help limit the size of the search space but they are not sufficient to determine a single correct route to the goal (for further detail see Howes, 1994).

Control knowledge determines which menu selections lead to the goal and which lead to dead-ends. It is acquired through exploration of the menu structure. However, exploration often makes control knowledge hard to acquire because the goal is temporally remote from the initial choices that must be made. For example, in Microsoft Word for the Apple Macintosh, if a user is trying to change a document from single to double columns their initial choice might be between "Format" and "View". If the selection is "Format", then the user will not know until after three further selections whether or not this choice was correct. In AYN working memory is bounded to store only the previous action. Thus, when the goal is achieved AYN only learns positive control knowledge for the selection immediately preceding the goal. On the next trial, when AYN reaches the selection known to be right (i.e. the one before the goal), it learns positive control knowledge for the immediately preceding selection that led to it. In this way positive knowledge is passed back up the structure in a final-first way until positive knowledge has been learnt for all the selections leading to the goal. AYN acquires negative control knowledge in a similar way for selections that lead to dead-ends.

The acquisition of recognition and control knowledge leads to two emergent behaviours: (1) over a number of trials the number of actions required to 
achieve the goal is reduced; (2) the model's reliance on recognition knowledge models the fact that users remain dependent on the display.

Whilst computer implementations of AYN successfully search and learn about menu structures, AYN itself remains largely untested. It is therefore clear that a more detailed empirical investigation is needed. The experiment reported in this paper was designed to test the following assumptions of the AYN model: reliance on recognition knowledge; the order of learning of correct actions at different levels within the menu tree (positive control knowledge); the learning of negative control knowledge.

In addition to testing AYN's assumptions, the experiment was also designed to examine the effect of extra information that might aid search (in this case, cues to level within the menu structure). Many researchers have studied the coarse-grained properties of various design heuristics (e.g. Mehlenbacher, Duffy and Palmer, 1989; Pierce, Parkinson and Sisson, 1992; Snowberry, Parkinson and Sisson, 1983) and also support tools (e.g. Howes and Payne, 1990; Van Hoe, Poupeye, Vandierendonck and De Soete, 1990; Webb and Kramer, 1990) for menu-based systems but these studies are not guided by a deep understanding of what the underlying cognitive mechanisms are. Instead, studies are designed to look for a measurable effect on a property such as overall task performance time. Unfortunately, this too frequently leads to nonsignificant results.

Level cues have been investigated previously, along with other search aids such as a trace of all previously selected options, a representation of the menu structure and a display of the entire previous menu (Van Hoe $e t$ al., 1990). None of these aids was found to affect the time taken to achieve the goal. However, this could be due to the fact that unlike many real-life menus, the experimental menus were too easy: the semantic relationship of the labels to the goal frequently determined the correct selection In situations where labels do not offer such clear guidance, search aids might have a greater effect on performance.

\subsection{Methodological issues}

Before we describe the experiment it is first necessary to discuss the problem of conducting experiments with menus with semantically unhelpful labels. The obvious problem in using semantically unhelpful labels rather than meaningful labels is that it changes the user's task. Thus, findings obtained with menu trees with semantically unhelpful labels might not apply to meaningful menus in actual devices. However, we argue that many menu labels in "real-world" devices are initially unhelpful to users despite the care taken in design. For example, a novice user of Microsoft Word for the Apple Macintosh might consider both the "Edit" and "Format" menus to be equally close in semantic terms to the goal of changing the text style. The task of choosing good menu labels may not be that much easier than the task of choosing good command-line words (cf. Furnas, Landauer, Gomez and Dumais, 1987).

One advantage of using menu structures with labels that give no semantic guidance to the user is that all subjects have the same prior semantic knowledge relevant to the task, namely none that can guide their choices. This avoids the problem of being unable to determine exactly what is influencing each decision in menus with meaningful labels where each subject will make selections on the basis of their prior semantic knowledge.

In the experiment presented here subjects were given menu structures in which the choices at each selection were pairs of words which were semantically unrelated to the goal but which were close semantic associates of each other (for example, "Pistol" and "Revolver" were the two choices at one node). The use of semantic pairs of words should ensure that the nodes are recognisable on subsequent visits, for example, the node with the options "Pistol" and "Revolver" might be recognised as a "gun" node.

\section{METHOD}

\subsection{Subjects}

32 undergraduate students from the Psychology Department at Cardiff University participated in this study for course credits.

\subsection{Materials and design}

Each subject was given four menu trees, each randomly generated from a set of 31 noun pairs. Each tree consisted of five levels with binary choices between a top and a bottom option at each node, as illustrated in Figure 1. A backup option was also provided at each node. The two options at a node were semantic noun pairs placed in alphabetical order (for example, "Carbon" and "Charcoal"). These noun pairs were carefully selected so that there was no overlap in meaning between any two pairs (both within and between trees). 


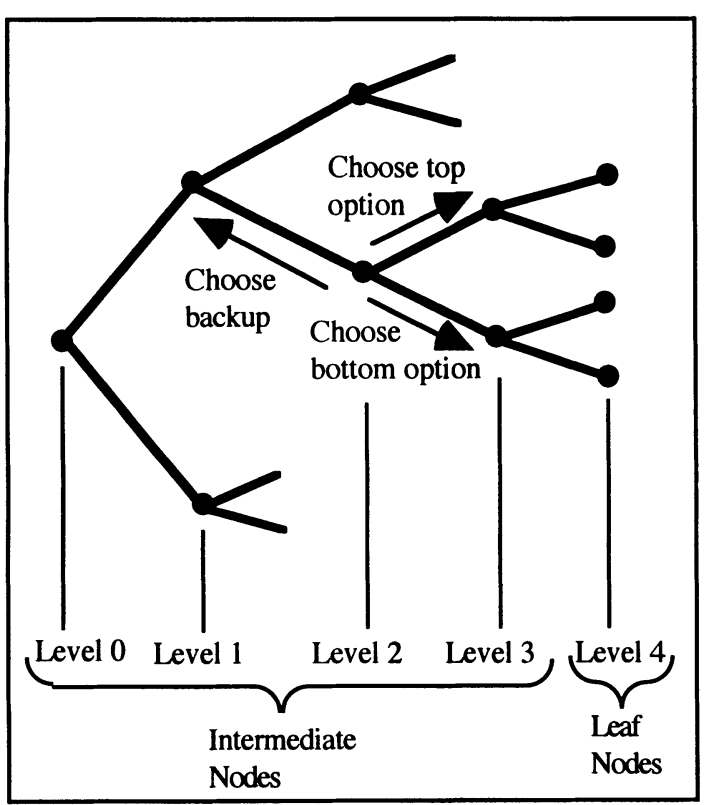

Figure 1. Partial illustration of the design of the trees used in the experiment.

One within-subjects factor was manipulated in this experiment. In addition the effect of practice on a number of variables was measured. The within-subjects factor concerned the presence or absence of a level cue. In two of the trees subjects were given a level cue which indicated the level of the current node within the tree (shown by a number between one - for the top level and four - for the lowest level). In the other two trees there was no cue to level. For half the subjects the level cue was present in the first and the third trees, for the other half the level cue was present in the second and the fourth trees.

Two other factors were manipulated in order to balance the design. The first of these was the order of presentation of the trees. Each subject was presented with the four different trees in one of four different presentation orders. These orders were based on a Balanced Latin Square to ensure that each tree was presented equally often before and after each of the others. The second factor that was manipulated was the order of presentation of the goals. All subjects had to search for the same four goals (one per tree) which were defined by their position in the menu (e.g. one goal was reached by first selecting the top option, then the top option, then the bottom option, then the bottom option and then the top option). At each level in the tree two of the goals required the top choice to be made and two required the bottom choice to be made. As for the trees, there were four presentation orders for the goals based on a Balanced Latin Square. Thus there were sixteen conditions in total formed by combining the four possible presentation orders for the goals and the trees.

\subsection{Procedure}

The experiment was presented on an Apple Macintosh computer using a program written in MacProlog32. Each subject was first shown the experimental instructions which contained information on the structure of the trees. Each subject was then given a practice task similar to the tasks used in the main part of the experiment. This practice task required the subject to search for a goal in a tree which had four levels (rather than the five levels used in the main part of the experiment). Level cues were present on the practice trial for all subjects. This should ensure that if any subject was unsure about the meaning of the level cues then s/he would ask about them during the practice trial and not during the experimental trials. After completing the practice task each subject was given the opportunity to review the instructions or to retry the practice task prior to starting the experimental trials.

The main part of the experiment consisted of ten trials. On each trial the subject was asked to search for the first goal in the first tree followed by the second goal in the second tree, and so on, as determined by the condition. The tasks were interleaved to prevent rehearsal from trial to trial. The choices made by the subject and the time taken to make each of those choices were automatically recorded by the program.

As outlined above, at each node in the tree, subjects were presented with a top and a bottom option together with a backup option. When one of the two options was selected the next choice point appeared. Selecting backup allowed subjects to backup to the previous node. It should be noted that the backup option was not present either in the top-level root node (where backup is not possible) or in the goal node (where backup would allow the subject to review the choices leading to the goal).

The duration of the experiment was between 45 minutes and one hour. 


\section{RESULTS}

\subsection{Total number of actions per task}

The total number of actions taken by each subject on each task on each trial was calculated. These data were subjected to an Anova to check for main effects of trial and level cue and for interactions between these variables. There was a significant main effect of trial, $\mathrm{F}(9,270)=87.429, p<0.01$ : the number of actions made decreased significantly over trials 1 to 4 , after which there were no significant changes. There was also a significant main effect of level cue, $F(1,30)=11.055$, $p<0.01$ : when a level cue was present subjects took significantly fewer actions (18.5 actions per task, on average) than when a level cue was absent ( 23.9 actions per task, on average). There was a significant interaction between trial and level cue, $F(9,270)=4.205$, $p<0.01$. The facilitatory effect of the level cue was only significant on trial 1 , thereafter there was no difference between the number of actions made when the level cue was present and absent.

\subsection{Percentage of correct actions taken on the success path}

For each node on the success path leading to the goal the percentage of correct actions made by subjects on their first visit to that node on each trial was calculated. The first visit to each node on each trial was used because the action taken on this visit should reflect the effects of long term memory rather than the effects of local, short-term search strategies. These data were subjected to an Anova to check for main effects of trial, level and level cue and for interactions between these variables.

There was a significant main effect of trial, $F(2$, $60)=168.04, p<0.01$ : the percentage of correct actions made increased with trials. In addition, there was a significant main effect of level, $\mathrm{F}(3,90)=4.15, p<0.01$ : a higher percentage of correct actions were made at level 0 (the top level in the tree) than at levels 1 or 3 (level 0 : $84.8 \%$ correct, level $1: 77.7 \%$ correct, level 2: $80.0 \%$ correct, level 3: $78.8 \%$ correct). There was no significant effect of level cue on success path actions.

There was a significant interaction between level and level cue, $\mathrm{F}(3,90)=5.170, p<0.01$. At level 3 , a significantly higher percentage of correct actions were made when there was no level cue than when a level cue was present. At all other levels the presence or absence of a level cue made no significant difference to the percentage of correct actions, as shown in Figure 2.

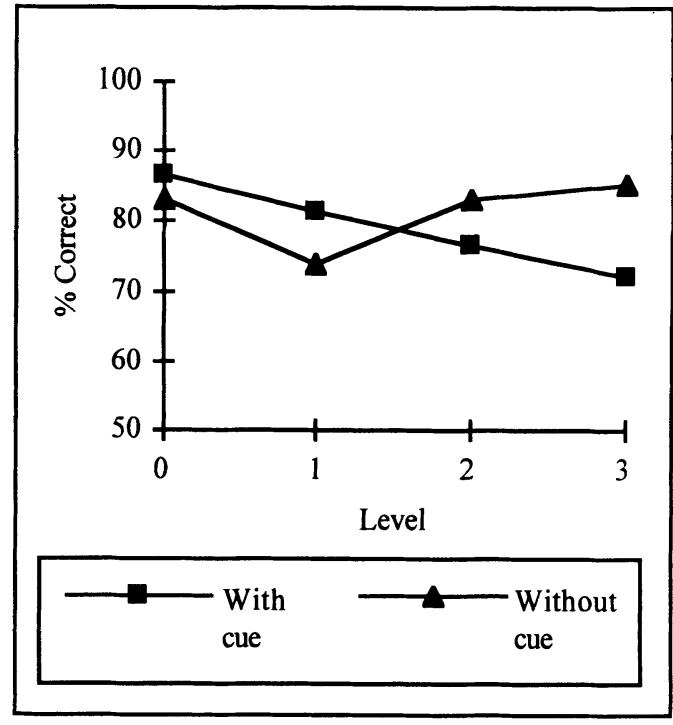

Figure 2. Graph showing mean percent correct at each level with and without a level cue present.

There was also a significant three-way interaction between trial group, level and level cue, $F(6$, $180)=4.531, p<0.01$. The interaction between level and level cue was significant only on trials $2-4$. On trials 5 10 there was no significant interaction between these two variables.

\subsection{Exhaustive search sequences}

The mean lengths of sequences of exhaustive and non-exhaustive search were calculated for each subject on each task on each trial. Exhaustive search was defined as consisting of only the following types of action: select either option at a node where neither option has been tried; select the untried option at a node where only one has been tried; select backup at a node where both options have been tried; select backup at a leaf node or a dead-end (where tried and untried actions were defined only in terms of the current search sequence - as soon as an exhaustive search sequence was broken, all actions were redefined as untried). In addition, in order to qualify as exhaustive search the following actions had to be included in the sequence: a back up from two tried actions, a visit to a leaf node.

These data were subjected to an Anova to test for main effects of trial, level cue and "exhaustiveness" and for interactions between these variables. There was a 
main effect of trial, $\mathrm{F}(9,270)=54.245, p<0.01$ : the length of both exhaustive and non-exhaustive search sequences decreased significantly over the first four trials but did not decrease further over trials 4 to 10 . There was a main effect of level cue, $F(1,30)=7.445$, $p=0.01$. Both exhaustive and non-exhaustive search sequences were shorter with a level cue (6.26 actions, on average) than without a level cue ( 7.53 actions, on average). There was also a main effect of exhaustiveness, $\mathrm{F}(1,30)=40.356, p<0.01$. Exhaustive search sequences were significantly shorter (4.31 actions, on average) than non-exhaustive search sequences (9.48 actions, on average).

On trial 1 alone, non-exhaustive search sequences were longer when there was not a level cue present than when a level cue was present. There was no effect of level cue on the length of exhaustive search sequences on trial 1 or any other trials: $\mathrm{F}(9,270)=3.874, p<0.01$, for the interaction between trial, level cue and exhaustiveness.

\subsection{Backups}

The percentage of actions that were backups at each node off the success path was calculated. These nodes were then grouped according to the number of previous forward moves made from them: no previous forward moves, between one and five previous forward moves, between six and ten previous forward moves or more than eleven previous forward moves. A forward move is one where the subject moves down the menu rather than backing up. The means are shown in Figure 3. Statistical analyses could not be carried out on these data due to the high number of missing data points.

On all trials it appears that more backups were made from nodes where there had been no previous forward visits than from nodes where there had been one or more previous forward visits.

On trial 3 only, more backups were made from nodes where there had been more than eleven forward visits than from nodes where there had been between one and ten previous forward visits. In addition, on trials 4 and 5 , more backups were made from nodes where there had been more than eleven forward visits than from nodes where there had only been between six and ten forward visits but not from nodes where there had been between one and five backups.

Over trials, the proportion of actions that were backups increased more rapidly for nodes where there had been no previous forward visits than for nodes where there had been one or more previous forward visits.

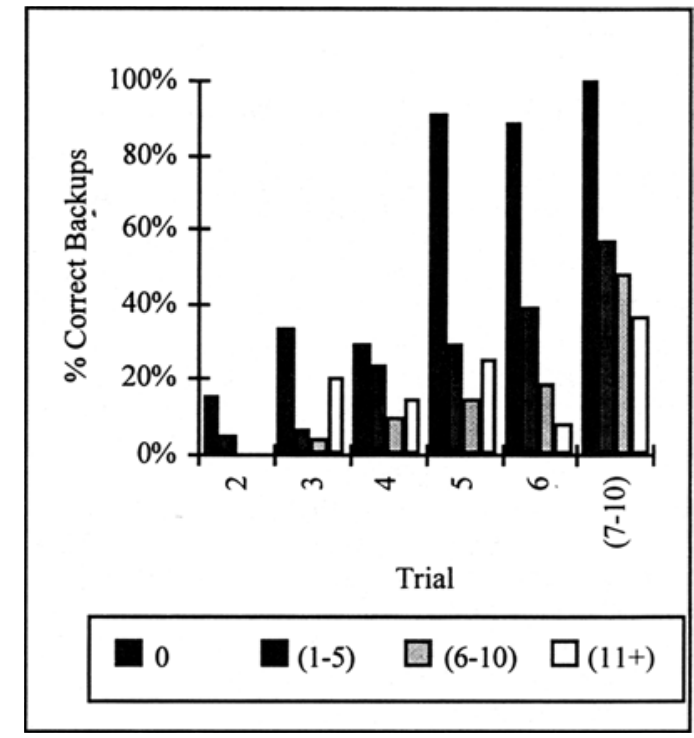

Figure 3. Graph showing the mean percentage of backup actions at each node according to the number of previous forward moves from that node.

\section{CONCLUSIONS AND DISCUSSION}

The main findings of the experiment were:

- When a level cue was present subjects took fewer actions to complete tasks on trial 1 . However, when a level cue was present subjects learnt the last action in a sequence more slowly than when there was no level cue.

- On trial 1 , non-exhaustive search sequences were longer when the level cue was absent than when it was present. There was no effect of level cue on the length of exhaustive search sequences.

- On all trials subjects were better at backing up from nodes where they had not previously made a forward move than from nodes where they had.

- Over trials, subjects got better at backing up from nodes where they had not previously made a forward move.

- On trials 3, 4 and 5 subjects were better at backing up from nodes where they had made eleven or more previous forward moves than from nodes where they had made between six and ten previous forward moves. 
As stated in the introduction, AYN uses recognition knowledge to help determine which option to choose at each selection point. A number of the results summarised above support this assumption of the model. In particular, subjects were better at backing up from nodes that they had not made a forward move from before than they were at backing up from nodes where they had made one or more previous forward moves. This indicates that visited and unvisited nodes are distinguished in subjects behaviour.

In addition, with practice subjects backed up from an increasing percentage of nodes from which they had not previously made a forward move. This improvement of recognition of nodes that have not been previously selected may occur for a similar reason to the reason for behaviour observed in IDXL (Rieman et al. 1996). The IDXL model selects the menu item that best matches the current task. In some cases this "best match" might be a previously ignored item at a higher node in the menu. Thus the IDXL model will choose to backup when an item at a higher node in the menu is a better match to the task than any of the choices at the currect node. In the case of the interactive search task, subjects might be making a similar relative judgement between the familiarity of the current node and the expected familiarity of a correct node and using this judgement as the basis for the decision whether to go forward from the node or whether to backup. Over trials the expected familiarity of the correct nodes will increase, consequently the difference in familiarity between correct and unvisited nodes will also increase and the relative judgement will become easier leading to an improvement in backing up from unvisited nodes. At present AYN does not account for relative familiarity judgements.

As well as using recognition knowledge AYN acquires control knowledge that determines both which actions lead to failure and which to success. The results do give some support to the hypothesis that subjects acquire negative knowledge. In particular, on trials 3, 4 and 5 subjects were more likely to backup from nodes where they had previously made more than eleven forward moves than from nodes where they had only made between six and ten forward moves. Whilst this is supportive, it also indicates that the learning appears not to be one-shot and instead requires a number of experiences. This is consistent with more gradual learning mechanisms (e.g. an instance theory of memory, Logan, 1988) than are embodied in AYN. However, it should be noted that on all trials subjects are much more likely to backup from nodes where there have been no forward moves whatsoever. This indicates that, if there is any learning of negative knowledge, then it contributes much less to performance than subjects' ability to recognise untried nodes does.

Whilst subjects clearly acquire knowledge of the correct route with practice, the data does not support the hypothesis that this route is acquired in a final-first order. Instead, all subjects seemed to learn the first action in the sequence before the others. AYN does not account for this result, and if anything would predict that the last action should be acquired first. Initial analyses not described above indicate that the result was not due to subjects visiting the first node more frequently than other nodes. However, the result may be due to a rehearsal strategy during initial search giving rise to a primacy effect. In addition, we note that learning the first choice first could be a rational strategy: (1) if a subject learns the correct selection to make at the first node in the menu tree then the space within which the goal must lie is halved; (2) the first node in the structure is a special case in that one of the two possible selections at this point must be on the route leading to the goal. This is true of no other node in the menu structure.

Finally, the presence or absence of a level cue had two main effects on performance.

(1) When a level cue was present subjects took fewer actions on the first trial to complete each task. This seems to be due to the fact that when there was a level cue, subjects' sequences of non-exhaustive search were shorter: the level cue seemed to reduce the lengths of the periods of time for which subjects searched unsystematically.

(2) Unexpectedly, when there was no level cue, subjects learned the last action of the route better than when there was a level cue. One possible explanation for this finding is that subjects might have been using the level cue to guide their exhaustive search rather than looking at the actual labels of the selections made. As a consequence, when there is no level cue subjects learn the labels of the selections leading to the goal. However, when a level cue is present the change in search strategy means that learning is disrupted.

Thus, overall the effect of providing level cues is not completely beneficial. Whilst the level cue helps initial exploration of the menu structure it appears to stop subjects from paying attention to the actual labels of the operators that they select.

In summary, through the initial, preliminary experiment reported here, we have moved towards 
developing an empirical understanding of how subjects remember routes through menus with labels that do not initially relate to the goal. Through practice subjects seem to acquire recognition knowledge and control knowledge that associates particular selections with the goal. In particular, we have tested some of the assumptions of the AYN model and found some supporting evidence and some challenges to its underlying mechanisms. We have also looked at the more applied issue of providing a level cue search aid and found that the effects are not all beneficial. We believe that these results are relevant to real-world interactive menu systems where the semantics of the menu labels do not determine a unique best selection.

Obviously, in order to develop a more detailed and complete account of interactive search, further work is required. In particular, we plan to follow two lines of future research: (1) Current work that we are undertaking investigates how subjects remember routes through menus which have some semantic structure. For example, it appears that "plausibility" probably interacts with the familiarity of menu items to determine subjects' choices. (2) In addition, we plan to investigate in more detail particular results obtained in this experiment, such as the order in which the correct choices are learnt, the effect of a level cue and the factors that lead subjects to make correct backups.

\section{ACKNOWLEDGEMENTS}

This work was supported by the ESRC Cognitive Engineering Initiative.

\section{REFERENCES}

Franzke, M. (1994) Exploration, acquisition and retention of skill with display-based systems. Ph.D. Thesis, Department of Psychology, University of Colorado, Boulder.

Franzke, M. (1995) Turning research into practice: characteristics of display-based interaction. Proceedings of the Conference on Human Factors in Computing Systems (New York, NY.), Association for Computing Machinery, 421-428.

Furnas, G.W., Landauer, T.K., Gomez, L.W., and Dumais, S.T. (1987) The vocabulary problem in humansystem communication. Communications of the $A C M$, 30, 11. 964-971.
Howes, A. (1994) A model of the Acquisition of Menu Knowledge by Exploration. In B. Adelson, S. Dumais and J. Olson (Eds.) Proceedings of Human Factors in Computing Systems CHI'94 (Boston, MA.), ACM Press, 445-451.

Howes, A. and Payne, S.J. (1990) Supporting exploratory learning. In D. Diaper, D. Gilmore, G. Cockton \& B. Shackel (Eds.), Human-Computer Interaction - INTERACT'90. Amsterdam: Elsevier Science Publishers, B.V. 881-885.

Kitajima, M. and Polson, P.G. (1995) A comprehension-based model of correct performance and errors in skilled, display-based, human-computer interaction. International Journal of Human-Computer Studies, 43, 65-69.

Logan, G.D. (1988) Toward an instance theory of automatization. Psychological Review, 95(4), 492-527.

Mehlenbacher, B., Duffy, T. M., and Palmer, J. (1989) Finding information on a menu: linking menu organisation to user's goals. Human-Computer Interaction, 4(3), 231-251.

Pierce, B.J., Parkinson, S.R., and Sisson, N. (1992) Effects of semantic similarity, omission probability and number of alternatives in computer menu search. International Journal of Man-Machine Studies, 37, 653677.

Rieman, J. (1994) Learning strategies and exploratory behaviour of interactive computer users. Ph.D. Thesis, Department of Computer Science, University of Colorado, Boulder.

Rieman, J., Young, R.M. and Howes, A. (1996) A dual-space model of iteratively deepening exploratory learning. International Journal of Human-Computer Studies, 44, 743-775.

Snowberry, K., Parkinson, S.K., and Sisson, N. (1983) Computer display menus. Ergonomics, 26(7), 699-712.

Van Hoe, R., Poupeye, K., Vandierendonck, A. and De Soete, G. (1990) Some Effect of Menu Characteristics and User Personality on Performance with Menu-Driven Interfaces. Behaviour and Information technology, 9 (1), 17-29.

Webb, J.M., and Kramer, A.F. (1990) Maps or analogies? A comparison of instructional aids for menu navigation. Human Factors, 32(3), 251-266. 